\title{
Figura ambígua e dislexia do desenvolvimento
}

\section{Ambiguous figure and developmental dyslexia}

Lílian Braga Alonso', Fabiana Maria Gomes Lamas², Paulo Ricardo Souza Sampaio ${ }^{3}$, José Ricardo Lima Rehder ${ }^{4}$

\section{Resumo}

Objetivo:Avaliar o processamento visual de figuras ambíguas em indivíduos disléxicos e compará-lo a um grupo controle leitor normal. Métodos: Survey comparativo onde foram selecionados aleatoriamente 39 escolares cursando entre a primeira e a quarta série do ensino fundamental. Deste total, 26 não apresentavam dificuldade de leitura ou mau rendimento escolar e constituíram o grupo controle. As outras 13 crianças (grupo de estudo) eram portadoras de Dislexia do desenvolvimento. Foram apresentadas, pela ordem, uma figura de Boring, uma figura com vasos de Rubin e uma figura pato/coelho de Jastrow. Resultados: Os resultados obtidos mostram que as crianças com diagnóstico de Dislexia do desenvolvimento perceberam número menor de figuras em relação às do grupo controle. Conclusão: O exame do processamento visual de crianças com Dislexia do desenvolvimento para figuras ambíguas é alterado quando comparado ao de crianças leitoras normais.

Descritores: Dislexia; Reconhecimento visual de modelos; Percepção visual

\footnotetext{
${ }^{1}$ Pedagoga, pós-graduanda lato sensu em distúrbios de aprendizagem do CRDA; pós-graduações e disciplina de Oftalmologia da Faculdade de Medicina do ABC - FMABC - Santo André (SP), Brasil;

${ }^{2}$ Pedagoga; Aluna de pós-graduação sensu stricto da Faculdade de Medicina do ABC - FMABC - Santo André (SP), Brasil;

${ }^{3}$ Chefe do Setor de Visão Subnormal - disciplina de Oftalmologia - Faculdade de Medicina do ABC - FMABC - Santo André (SP), Brasil;

${ }^{4}$ Professor Titular de Oftalmologia - Faculdade de Medicina do ABC - FMABC - Santo André (SP), Brasil.

Trabalho foi realizado na disciplina de Oftalmologia - Faculdade de Medicina do ABC - FMABC - Santo André (SP), Brasil.
}

Recebido para publicação em: 25/1/2008 - Aceito para publicação em 28/3/2008 


\section{INTRODUÇÃO}

$\mathbf{O}$ termo ambíguo deriva do latim ambigùus, a,um 'que tem dois sentidos, equívoco, duvidoso' ${ }^{\text {(1) }}$. O conceito de figura ambígua, também conhecida como figura-fundo foi desenvolvido pela Psicologia da Forma (Gestalt), e tenta explicar como percebemos figuras definidas e salientadas que ficam em fundos indefinidos ${ }^{(2)}$.

Entre as chamadas figuras ambíguas mais famosas estão: a figura de Eduards Boring (velha ou jovem?), os vasos de Rubin ${ }^{(3)}$ (ora percebe uma taça ora duas faces uma defronte a outra), a figura pato/coelho de Jastrow $^{(4)}$, e os cubos de Necker ${ }^{(5)}$.

Estudos eletrofisiológicos e trabalhos de neuroimagem descrevem padrões diferenciados de ativação do córtex occipto-temporal do cérebro humano quando um indivíduo observa figuras ambíguas ${ }^{(3,6-7)}$.

A Dislexia do desenvolvimento é caracterizada pela dificuldade no processamento da leitura. Outros estudos eletrofisiológicos e trabalhos de neuroimagem mostram alterações na ativação do córtex temporo-parietal do cérebro humano em indivíduos disléxicos ${ }^{(9-11)}$.

Indivíduos disléxicos mostram, também, alterações nos testes de figura-fundo auditiva ${ }^{(12)}$.

O que motivou a realização do presente estudo preliminar foi o fato de que, na literatura pesquisada, não encontramos nenhum trabalho científico que mostre a percepção de um portador de Dislexia do desenvolvimento frente a uma figura ambígua.

\section{Овjetivo}

Conhecer a percepção de leitores normais e de portadores de Dislexia do desenvolvimento durante a observação de figuras ambíguas.

\section{Métodos}

Tipo de estudo: survey comparativo.

População e amostra: foram selecionados, aleatoriamente, 39 escolares cursando entre a primeira e a quarta série do ensino fundamental. Deste total, 26 não apresentavam dificuldade de leitura ou mau rendimento escolar e constituíram o grupo controle. As outras 13 crianças (grupo de estudo) eram portadoras de Dislexia do desenvolvimento com diagnóstico firmado pela equipe multiprofissional do Centro de Referência em Distúrbios de Aprendizagem em São Paulo - Brasil.
Critérios de inclusão

- escolaridade entre a primeira e quarta série do ensino fundamental;

- idade cronológica entre 7 e 10 anos completos;

- prévia avaliação oftalmológica e ortóptica completa;

- diagnóstico firmado de Dislexia do desenvolvimento sem alterações psicológicas ou comportamentais. Critérios de exclusão

- uso recente de medicamentos de qualquer tipo

- uso de óculos com lentes especiais.

Técnica empregada

Duas professoras do ensino fundamental, especializadas no reconhecimento de crianças portadoras de distúrbios de aprendizagem, apresentaram, na seqüência, uma figura de Boring, uma figura com vasos de Rubin e uma figura pato/coelho de Jastrow. As figuras estavam impressas em papel A4 na cor preta e sem plastificação. Cada imagem percebida totalizava um ponto. A somatória das imagens das 3 figuras resultaria em 6 pontos. Imagens inexistentes e informadas não contariam pontos. A criança recebia informações sobre o exame e a seguir era entregue uma folha de cada vez com a figura a ser testada. Nenhum outro auxílio era concedido durante o exame. Os indivíduos que necessitavam correção óptica (óculos para miopia, astigmatismo e hipermetropia) deveriam usá-lo continuamente há mais de 3 meses e também deveriam usá-lo durante a avaliação.

Variáveis dependentes

Número total de imagens percebidas pela criança nas 3 figuras apresentadas.

Variáveis independentes

Tiveram somente conotação descritiva.

\section{Resultados}

Nos dois grupos a distribuição quanto ao sexo foi de $50 \%$ para crianças de sexo masculino e $50 \%$ para crianças do sexo feminino.

A média das idades para o grupo controle foi de 8,6 anos e de 8,5 anos para o grupo de estudo.

Nos dois grupos $25 \%$ dos escolares usavam óculos para correção de algum vício de refração.

Nenhum dos avaliados informou conhecimento prévio das figuras apresentadas.

A tabela 1 apresenta o número médio de imagens informadas nos dois grupos e a porcentagem obtida em relação ao total previsto de imagens apresentadas (6 imagens). 
Tabela 1

Número médio de imagens informadas nos dois grupos e a porcentagem obtida em relação ao total previsto de imagens apresentadas (6 imagens)

\begin{tabular}{lcccccc}
\hline & \multicolumn{3}{c}{ GRUPO ESTUDO } & \multicolumn{3}{c}{ GRUPO CONTROLE } \\
\hline Escolaridade & Crianças & Figuras & $\%$ & Crianças & Figuras & $\%$ \\
\hline $1^{\text {a }}$ Série & 3 & 0 & 0 & 6 & 3 & 50 \\
$2^{\text {a }}$ Série & 3 & 1 & 16,7 & 6 & 4 & 66,7 \\
$3^{\text {a }}$ Série & 3 & 2 & 33,3 & 6 & 4 & 66,7 \\
$4^{\text {a }}$ Série & 4 & 2 & 33,3 & 8 & 5 & 83,3 \\
Total & 13 & - & - & 26 & - & - \\
\hline
\end{tabular}

\section{DISCUSSÃO}

Os estudos que relacionam a função visual e a Dislexia do desenvolvimento mostram controvérsias. Enquanto alguns autores não reconhecem a existência de alterações na percepção visual destes pacientes ${ }^{(13)}$ outros informam insuficiência de convergência, simultanagnosia e deficit de percepção visoespacial ${ }^{(1415)}$.

Em nossa avaliação, $50 \%$ dos indivíduos portadores de Dislexia do desenvolvimento apresentavam insuficiência de convergência, mesmo com o uso de lentes corretivas para o vício de refração.

Surge a pergunta se estas crianças apresentariam melhor desempenho se tivessem sido previamente submetidos a exercícios de ortóptica. Novos trabalhos devem ser realizados com esse objetivo.

Define-se simultanagnosia como a habilidade do indivíduo em reconhecer múltiplos elementos em uma cena visual apresentada. Esta habilidade pode ser importante para a percepção da figura-fundo e também não foi previamente testada nos grupos controle e estudo. Novas pesquisas deverão ser realizadas com este fim.

A escolha da instituição responsável pelo diagnóstico dos portadores de Dislexia do desenvolvimento ocorreu em virtude do protocolo meticuloso utilizado por aquela instituição. Os indivíduos ali examinados são submetidos à avaliação neuropediátrica completa (inclusive com exame neuroevolutivo, mapeamento cerebral,TAVIS, eletroencefalografia e ressonância magnética), testes psicopedagógicos, fonoaudiológicos, psicológicos e oftalmológicos.

\section{Conclusão}

Os resultados obtidos mostram que as crianças com diagnóstico de Dislexia do desenvolvimento per- ceberam número menor de figuras em relação às do grupo controle. Trabalhos complementares devem ser realizados para estudar melhor este problema.

\section{Abstracts}

Objective: Evaluate the visual response of children with developmental dyslexia when ambiguous figure was presented in comparison with normal readers children. Methods: 39 children from basic school was randomly chosen. 26 were normal readers. 13 were diagnosed developmental dyslexia. Were presented the Boring figure, Rubin vessels figure and Jastrow figure. Results: Participants in the clinical group with dyslexia performed marginally less well than those in the control group. Conclusion: The visual response of children with developmental dyslexia when ambiguous figure was presented is less well in comparison with normal readers children

Keywords: Dyslexia; Pattern Recognition, Visual; Visual perception

\section{ReFERÊNCIAS}

1. HOUAISS Dicionário Eletrônico da Língua Portuguesa [CDROM]. versão 1.0.7. São Paulo: Objetiva; 2004

2. Zanker JM, Abdullah AA. Are size illusions in simple line drawings affected by shading? Perception. 33(12):1475-82, 2004.

3. Hasson U, Hendler T, Ben Bashat D, Malach R. Vase or face? A neural correlate of shape-selective grouping processes in the human brain. J Cogn Neurosci. 2001;13(6):744-53.

4. Brugger P. One hundred years of an ambiguous figure: happy birthday, duck/rabbit.Percept Mot Skills. 1999 Dec;89(3 Pt 1):973-7.

5. Kornmeier J, Bach M. The Necker cube an-ambiguous figure disambiguated in early visual processing. Vision Res. 2005;45(8):955-60. 
6. Long GM, Moran CJ. How to keep a reversible figure from reversing: teasing out top-down and bottom-up processes. Perception. 2007;36(3):431-45.

7. Kornmeier J, Ehm W, Bigalke H, Bach M. Discontinuous presentation of ambiguous figures: how interstimulus-interval durations affect reversal dynamics and ERPs. Psychophysiology. 2007;44(4):552-60.

8. Johansson BB. Cultural and linguistic influence on brain organization for language and possible consequences for dyslexia: a review. Ann Dyslexia. 2006;56(1):13-50.

9. Barnes J, Hinkley L, Masters S, Boubert L. Visual memory transformations in dyslexia.Percept Mot Skills. 2007;104(3 Pt 1):881-91.

10. Hoeft F, Meyler A, Hernandez A, Juel C, Taylor-Hill H, Martindale JL, et al. Functional and morphometric brain dissociation between dyslexia and reading ability.Proc Natl Acad Sci U S A. 2007;104(10):4234-9.

11. Arduini RG, Capellini SA, Ciasca SM. Comparative study of the neuropsychological and neuroimaging evaluations in children with dyslexia. Arq Neuropsiquiatr. 2006;64(2B):369-75.

12. Frota $\mathrm{S}$. Auditory processing: study in children with specific reading and writing deficits [tese]. São Paulo; Escola Paulista de Medicina da Universidade Federal de São Paulo; 2003.
13. Pache M, Weber P, Klumpp S, Gutzwiller P, Kaiser HJ. [Visual function in developmental dyslexia. Opthalmological and neuropsychological results] Ophthalmologe. 2004;101(9):90713. German.

14. Quercia P, Seigneuric A, Chariot S, Vernet P, Pozzo T, Bron A, Creuzot-Garcher C, Robichon F. [Ocular proprioception and developmental dyslexia. Sixty clinical observations] J Fr Ophtalmol. 2005;28(7):713-23. French.

15. Sampaio P. Contribution to the study of developmental dyslexia: the reading performance with prismatic lenses [tese]. São Paulo: Faculdade de Medicina da Universidade de São Paulo; 2003.
ENDEREÇO PARA CORRESPONDÊNCIA:
Paulo Ricardo Souza Sampaio
Rua Correa Dias, 184 - cj 55
CEP 04104-000 - São Paulo - SP
E-mail: paulosampaio@dislexia.med.br
Telefax: (11) 5575-3087 